\title{
Thermal, spectroscopic, and dissolution studies of the simvastatin-acetylsalicylic acid mixtures
}

\author{
Agata Górniak • Bożena Karolewicz • \\ Ewa Żurawska-Płaksej · Janusz Pluta
}

Received: 22 June 2012/ Accepted: 13 August 2012/Published online: 8 September 2012

(C) The Author(s) 2012. This article is published with open access at Springerlink.com

\begin{abstract}
The objective of the present investigation was to study the effect of eutectic formation on in vitro dissolution of simvastatin (SIM) released from mixtures with acetylsalicylic acid (ASA) prepared by a grinding method. SIMASA mixtures were characterized by means of differential scanning calorimetry (DSC), infrared spectroscopy (IR), $\mathrm{X}$-ray powder diffractometry (XRPD), and in vitro dissolution tests. IR spectroscopy and XRPD studies indicated no interaction between SIM and ASA in the solid state. The DSC investigation has revealed that SIM and ASA form a simple eutectic system containing $66.6 \% \mathrm{w} / \mathrm{w}$ of SIM at the eutectic point. In vitro dissolution studies of SIM and its mixtures with ASA were carried out. The eutectic mixture shows an appreciable increase in the dissolution rate in comparison with other ratios and SIM in $0.5 \% \mathrm{w} / \mathrm{v}$ sodium lauryl sulfate. The dissolution enhancement of SIM was related to the effective wetting of the drug particles with a significantly reduced size released from eutectic composition. In conclusion, dissolution of SIM can be enhanced through eutectic formation with ASA by means of simple mechanical activation (a grinding method).
\end{abstract}

\footnotetext{
A. Górniak ( $₫)$

Department of Inorganic Chemistry, Wroclaw Medical

University, Szewska 38, 50-139 Wrocław, Poland

e-mail: agata.gorniak@interia.pl

B. Karolewicz $\cdot$ J. Pluta

Department of Drug Form Technology, Wroclaw Medical

University, Szewska 38, 50-139 Wrocław, Poland

E. Żurawska-Płaksej

Department of Pharmaceutical Biochemistry, Wroclaw Medical

University, Szewska 38, 50-139 Wrocław, Poland
}

Keywords Phase diagram - Eutectic - Simvastatin . Aspirin - Dissolution enhancement - Solid dispersion

\section{Introduction}

Patients suffering from cardiovascular disease are usually treated with a simultaneous administration of cholesterollowering drugs, antithrombotic (aspirin), and $\beta$ blockers. The idea of combining two or more drugs with a complementary mode of action is to obtain an additivity of the desired therapeutic effect. Pharmaceutical preparations containing multiple active ingredients are essentially used to assure patient compliance and play an important role in the development of drug delivery systems [1].

Simvastatin (SIM) is a competitive inhibitor of 3-hydroxy-3-methylglutaryl-coenzyme A reductase and belongs to the group of drugs called statins, widely used for the treatment of hypercholesterolemia [2]. Statins beyond the effects of lowering the serum cholesterol levels have many pleiotropic effects manifested among others through the protective effects on the vascular endothelium, plaque stabilization, anti-inflammatory effects, and the impact on the coagulation system and fibrinolysis [3-6].

Acetylsalicylic acid (ASA), also known as aspirin, has been used as an analgesic, an antipyretic, and an antiinflammatory nonsteroidal drug (agent). Since the time when it was indicated that a low-dose ASA revealed an inhibiting effect on platelet aggregation, aspirin has been applied to prevent complications of atherosclerotic cardiovascular disease such as myocardial infarction and stroke [7, 8].

A combination therapy of aspirin and statin plays an important role in the development of an improved drug delivery system for primary and secondary prevention of coronary heart disease, ischemic stroke, and vascular 
prevention of complications in diabetic patients, and may produce synergy and has additive effects on the immune response to inflammation. Coadministration of statins and aspirin reduces the incidence of cardiovascular disease and improves blood circulation in the blood vessels. Both substances inhibit platelet function, resulting in the inhibition of thromboxane A2, which has pro-aggregation and angiospastic activity [9]. Trials by Hennekens et al. [10] showed that the aspirin therapy provided an additive effect of $24 \%$ relative risk reduction of cardiovascular events in addition to a statin monotherapy. The statin therapy provided an additional $13 \%$ relative risk reduction to an aspirin monotherapy [10]. A recent study demonstrated that the treatment with the combined therapy reduced the extent of inflammation as compared to the treatment with a SIM or aspirin monotherapy, indicating that the aspirin SIM combination has a synergistic effect concerning the immune response to the inflammatory challenge manifested in the lower concentration of the inflammation biomarkers [11, 12].

For orally administered drugs, solubility is one of the most limiting parameters affecting their appropriate concentration in systemic circulation and in consequence the desired pharmacological effect [13]. SIM is the most lipophilic statin [14], practically insoluble in water $\left(30 \mu \mathrm{g} \mathrm{mL}^{-1}\right)$ [15]. The oral bioavailability of the active form of SIM is $5 \%$, which is due to extensive first pass hepatic metabolism [16]. The acceptable permeability through biomembranes and the low solubility places SIM in class II of the Biopharmaceutics Classification System. Therefore, solubility improvement of SIM may lead to the enhancement of bioavailability and the pharmacological response.

Several approaches have been employed to improve the solubility of SIM such as the preparation of spherically agglomerated crystals of the drug [17], solid dispersions with PEG 6000 [18, 19], PEG 4000 [19, 20], PVP K30 [20, 21], poloxamer 188 [21], or preparation of inclusion complexes with $\beta$-cyclodextrin [22] and hydroxypropyl- $\beta$ cyclodextrin [19, 23].

A promising method to enhance solubility is to obtain a drug-drug eutectic mixture, which belongs to the crystalline solid dispersion group (first generation solid dispersions) $[24,25]$. Combining the two substances in a binary eutectic system influences the decrease of the melting point of the mixture, which facilitates their permeation through the skin [26, 27]. At the eutectic point (composition), there is a direct phase transition of all components from the solid into the liquid phase. The main advantages of eutectic mixtures for the achievement of improved dissolution is that the drug particles will be solidified in their thermodynamically stable crystal form so conversion upon storage is not an issue and the solidification process produces an intimate mixture of fine crystals.

The aim of our study was to evaluate the impact of the eutectic formation in the binary system SIM-ASA on the dissolution of the examined drugs in aqueous solution. Differential scanning calorimetry (DSC) is a widely and effectively used thermal method for solid-state characterization in pharmaceutical preformulation, i.e., eutectic and solid solution formation [28], detection and identification of polymorphic form [29, 30], inclusion complexation [31], and compatibility investigation $[32,33]$. We used DSC, as the main method to investigate the phase equilibrium of that binary system, the knowledge of which is required to determine a eutectic composition. An in vitro dissolution study was carried out to evaluate the drug release rate from the examined mixtures. Furthermore, we used infrared spectroscopy (IR) and X-ray powder diffraction (XRPD) to evaluate the physicochemical properties of the obtained binary mixtures and to study the possible interactions between the examined drugs.

\section{Experimental}

\section{Materials}

SIM was donated by Polpharma SA (Poland). ASA with an assay of $99 \%$ and phosphoric acid were supplied from Aldrich. Sodium lauryl sulfate (SLS) was purchased from P.P.H. "Stanlab" (Poland). Potassium bromide was obtained from POCH (Poland). Acetonitrile HPLC grade was obtained from J. T. Baker (The Netherlands).

\section{Preparation of mixtures}

Due to the risk of aspirin decomposition during the melting, a simple method of grinding was utilized to obtain SIM-ASA solid dispersion. Finely powdered mixtures were prepared from accurately weighed quantities of each component and very thoroughly ground together for at least 5 min using an agate mortar and pestle. Samples were weighed using a Mettler Toledo AT 261 microbalance $( \pm 0.01 \mathrm{mg})$. Fourteen binary mixtures of SIM and ASA were prepared. The weight ratios for the SIM-ASA mixtures were 95.0/5.0, 89.8/10.2, 85.0/15.0, 80.0/20.0, 70.0/ $30.0,66.6 / 33.4,59.8 / 40.2,55.0 / 45.0,50.0 / 50.0,40.0 / 60.0$, $30.3 / 69.7,20.0 / 80.0,12.6 / 87.4$, and 5.0/95.0 \%. Mixtures were sieved through a $355-\mu \mathrm{m}$ mesh size and were stored in a desiccator over calcium chloride.

\section{Differential scanning calorimetry (DSC)}

The DSC curves of each mixture were obtained using a Mettler Toledo DSC 25 measuring cell equipped with a heat flow sensor and joined via interface TA Controller TC 15 to a computer. The measurements were driven by STAR $^{\mathrm{e}}$ software. 
Samples for DSC measurements were sealed in $40-\mu 1$ standard aluminum crucibles with a single hole punched in the lid. An empty pan of the same type was employed as a reference. The DSC instrument was calibrated with the melting point of indium $\left(156.6{ }^{\circ} \mathrm{C}\right)$ as a standard $[34,35]$. DSC scans of each mixture were performed at a heating rate of $5{ }^{\circ} \mathrm{C} \min ^{-1}$ in the temperature range of $30-170{ }^{\circ} \mathrm{C}$. The DSC cell was purged with a stream of dry argon at a rate of $50 \mathrm{~cm}^{3} \mathrm{~min}^{-1}$. Experiments were performed in triplicate and the mean values were calculated.

\section{$\mathrm{X}$-ray powder diffractometry}

Powder diffraction patterns of all samples were performed over the range $2 \theta$ from $5^{\circ}$ to $40^{\circ}$, at steps of $0.04^{\circ}$ and a counting time of $1.5-3^{\circ} \mathrm{min}^{-1}$, using a Siemens D5000 powder diffractometer with $\mathrm{CuK} \alpha$ and a Ni-filtered radiation $(\lambda=0.15405 \mathrm{~nm})$.

IR spectroscopy

IR spectra were obtained using IR-Specord M80 (CarlZeiss-Jena, Germany). Samples were mixed with potassium bromide (spectroscopic grade) and compressed into a disk using a hydraulic press (Specac, USA) before scanning from 4000 to $200 \mathrm{~cm}^{-1}$. The spectrum of a $\mathrm{KBr}$ pellet was used as the background.

In vitro dissolution studies

The in vitro dissolution studies of capsules containing SIM, ASA, and their mixtures were carried out in triplicate on an apparatus 1 (baskets). A VanKel VK 7025 apparatus was used. Before the start of the dissolution test, the hard gelatin capsules $(0.95 \mathrm{~mL})$ were tested in terms of disintegration time in the dissolution medium. Their effect on the obtained dissolution rate was found to be negligible. The $100 \mathrm{mg}$ of each of the prepared mixtures as well as reference substances- $66.6 \mathrm{mg}$ of SIM and $100 \mathrm{mg}$ of ASA were transferred into capsules immediately before the dissolution test. The dissolution studies were performed in $500 \mathrm{~mL}$ of $0.5 \%(\mathrm{w} / \mathrm{v})$ SLS aqueous solution at $37 \pm 0.5{ }^{\circ} \mathrm{C}$ and $50 \mathrm{rpm}$. Samples were collected at 5, 10, $15,20,25,30,35,40,50$, and $60 \mathrm{~min}$ using an automatic sampler, and filtered $(0.20-\mu \mathrm{m}$ pore size $)$ and analyzed immediately after sampling. The quantitative determination for SIM and ASA was performed with HPLC system with a VWD (Agilent 1100). The analysis was carried out using a Zorbax ODS $\mathrm{C}_{18}$ column $(5 \mu \mathrm{m}, 4.6 \mathrm{~mm} \times$ $150 \mathrm{~mm}$, Agilent). The terms of the analysis were based on a detailed monograph on SIM included in the Polish Pharmacopoeia VIII [36]. The analysis was performed by combined isocratic and gradient elutions with a mixture of solvents on the composition of acetonitrile-water (with $0.1 \%$ phosphoric acid) in different ratios with a steady flow rate of $2 \mathrm{~mL} \mathrm{~min}{ }^{-1}$. The substances eluted from the column were identified by a spectrophotometric detector at $238 \mathrm{~nm}$. The peak appearing on the chromatogram in the second minute was characteristic of ASA, whereas the retention time for SIM was 5 min (Fig. 1). Peak area data as a function of concentration of the examined compounds were collected by ChemStation software supplied by Agilent. External standards of SIM and ASA were used to obtain calibration curves. The evaluation methods were linear in the range of $20-250 \mu \mathrm{g} \mathrm{mL}^{-1}$ for both compounds (linearity $\left.r^{2}=0.999\right)$. Dissolution experiments were performed in triplicate and the mean values were calculated.

\section{Results and discussion}

Thermal studies

The DSC curves of the various mixtures clearly indicated that the investigated compounds formed a binary eutectic system. The phase diagram constructed on the basis of the DSC results is presented in Fig. 2. The DSC scans are typical of eutectic systems. The first peak consistently appeared near $105.6{ }^{\circ} \mathrm{C}$, indicating the eutectic reaction. This temperature is also known as the temperature of solidus on a phase diagram. The second peak (temperature of liquidus) was generally wider, indicating that complete melting took place over a temperature range. Only these two kinds of thermal effects are shown by DSC curves for the whole range of compositions. The melting points of the pure components (ASA: $141.9^{\circ} \mathrm{C}$; SIM: $139.5^{\circ} \mathrm{C}$ ) were depressed due to the existence of the other component in the mixture as shown in the phase diagram in Fig. 2. The examples of DSC curves showing both eutectic and liquidus events are presented in Fig. 3. It should be added that DSC curves of pure ASA as well as those of pure SIM have shown sharp melting endotherms, which permitted the determination of the respective melting points precisely.

The onset of the first peak occurs approximately at the same temperature of $105.6{ }^{\circ} \mathrm{C}$ for all the compositions and represents the eutectic reaction:

$$
\begin{aligned}
& \text { Solid acetylsalicylic acid (ASA) } \\
& \quad+\operatorname{solid} \operatorname{simvastatin}(\text { SIM })=\operatorname{liquid}(\mathrm{L}) .
\end{aligned}
$$

For this type of transition, the peak position does not vary with the content of a component, whereas the heat of the thermal events rises when approaching the eutectic point. In this work, the thermal effect corresponding to the eutectic reaction was noticeable on DSC curves for most mixtures examined (Fig. 3). Values of the eutectic melting enthalpy $\Delta H\left(\mathrm{~kJ} \mathrm{~mol}^{-1}\right)$ for a given mixture, determined 
Fig. 1 Chromatogram of 5.0/ $95.0 \% \mathrm{w} / \mathrm{w}$ SIM/ASA mixture

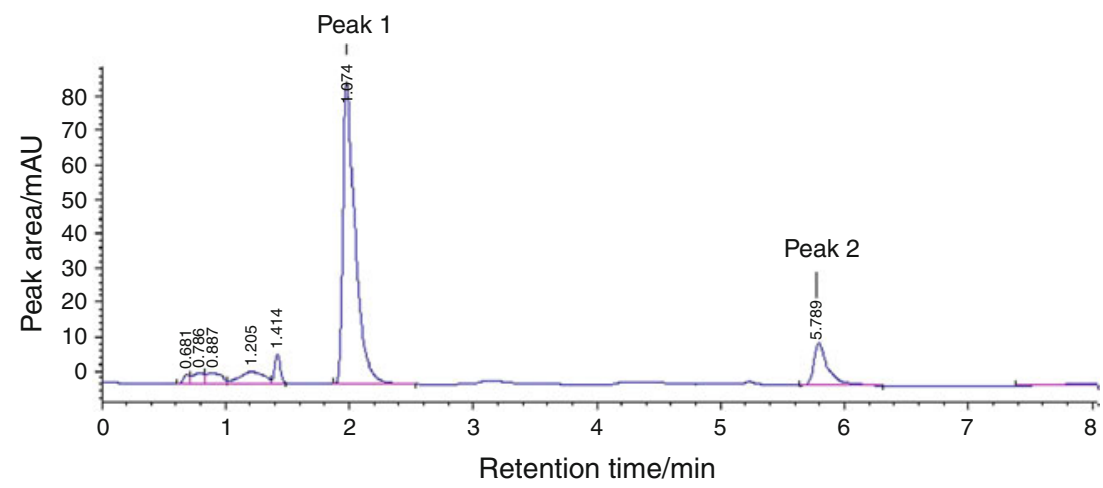

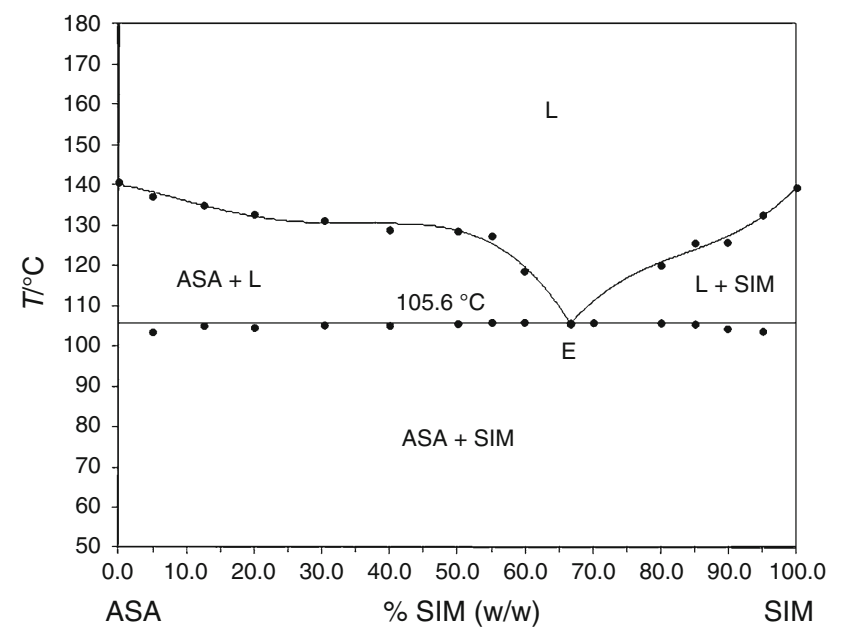

Fig. 2 Phase equilibrium diagram of the investigated system SIM-ASA

by integration of the eutectic peak area on DSC curves, are plotted in Fig. 4 versus weight fraction of SIM (Tamman's triangle construction). The maximum enthalpy value has been found for $66.6 \% \mathrm{w} / \mathrm{w}$ of SIM which corresponds to the composition of the eutectic point and is in agreement with the phase diagram (Fig. 2). The thermal effect of the eutectic transition goes to zero for compositions corresponding to pure components. This confirms that there is no mutual miscibility in the solid state and no formation of terminal solid solutions at either side of the phase diagram. Finally, parameters of the eutectic point $(E)$ have been established as follows:

- composition: weight fraction of SIM $66.6 \%$, weight fraction of ASA $33.4 \%$;

- temperature: $105.6^{\circ} \mathrm{C}$.

Spectroscopic studies

$X$-ray powder diffractometry

The obtained XRPD patterns of SIM, ASA, and their mixtures are shown in Fig. 5. The diffraction pattern of the SIM shows a crystalline nature of the drug, indicated by numerous distinctive peaks at a diffraction angle $(2 \theta)$ of $9.28^{\circ}, 10.84^{\circ}$, $14.88^{\circ}, 15.56^{\circ}, 16.48^{\circ}, 17.16^{\circ}, 17.64^{\circ}, 18.72^{\circ}, 19.32^{\circ}$, and $22.48^{\circ}$. The drug's crystalline peaks were still detectable in the mixture with ASA. The X-ray diffractogram of ASA shows characteristic distinctive sharp peaks at diffraction angles $(2 \theta)$ of $7.76^{\circ}$ and $15.60^{\circ}$. The XRPD pattern for the sample containing $95 \% \mathrm{w} / \mathrm{w}$ SIM shows the presence of a slight amount of the second phase (ASA), which can be deduced from the appearance of a very weak signal at $2 \theta 27^{\circ}$ (distinctive ASA peak) and evidenced by a higher SIM peak at $2 \theta 15.56^{\circ}$. The latter effect is caused by the superposition of the line of ASA at $2 \theta 15.60^{\circ}$, one of the most intensive in the ASA spectrum. On the other hand, the XRPD pattern of the sample containing $95 \% \mathrm{w} / \mathrm{w}$ ASA shows the presence of a slight amount of crystalline SIM, which can be identified by the presence of weak SIM peaks at $2 \theta 9.3^{\circ}$ and $17.16^{\circ}$. These data confirmed that at room temperature, the two components of which investigated solid dispersions are formed do not create solid solutions. Moreover, no other peaks than those which could be assigned to pure SIM and ASA were detected on diffractograms registered for solid dispersions, indicating no chemical interactions in the solid state between the two entities.

\section{IR spectroscopy}

IR spectra of SIM, ASA, and 19.4/80.6\% w/w SIM/ASA, $89.8 / 10.2 \%$ w/w SIM/ASA are shown in Fig. 6 . There is no significant difference in the IR spectra of pure drugs and their mixtures. All major SIM peaks observed at wave numbers $3553 \mathrm{~cm}^{-1}$ (free O-H stretching vibrations), 3011, 2959, and $2872 \mathrm{~cm}^{-1}$ (methyl C-H asymmetric stretching vibrations), $1714 \mathrm{~cm}^{-1}$ (stretching vibrations of ester and lactones carbonyl functional groups), absorption bands characteristic of ASA at $1605 \mathrm{~cm}^{-1}$ (benzene ring stretching vibrations), 1680 and $1750 \mathrm{~cm}^{-1}$ (vibrations of aliphatic carbonyls), and a broader band at $2500-3500 \mathrm{~cm}^{-1}$ (stretching vibrations of carboxylic acid hydroxyl group) were retained in mixtures, which clearly indicates no interaction between the drugs. 
Fig. 3 DSC thermograms of SIM-ASA mixtures
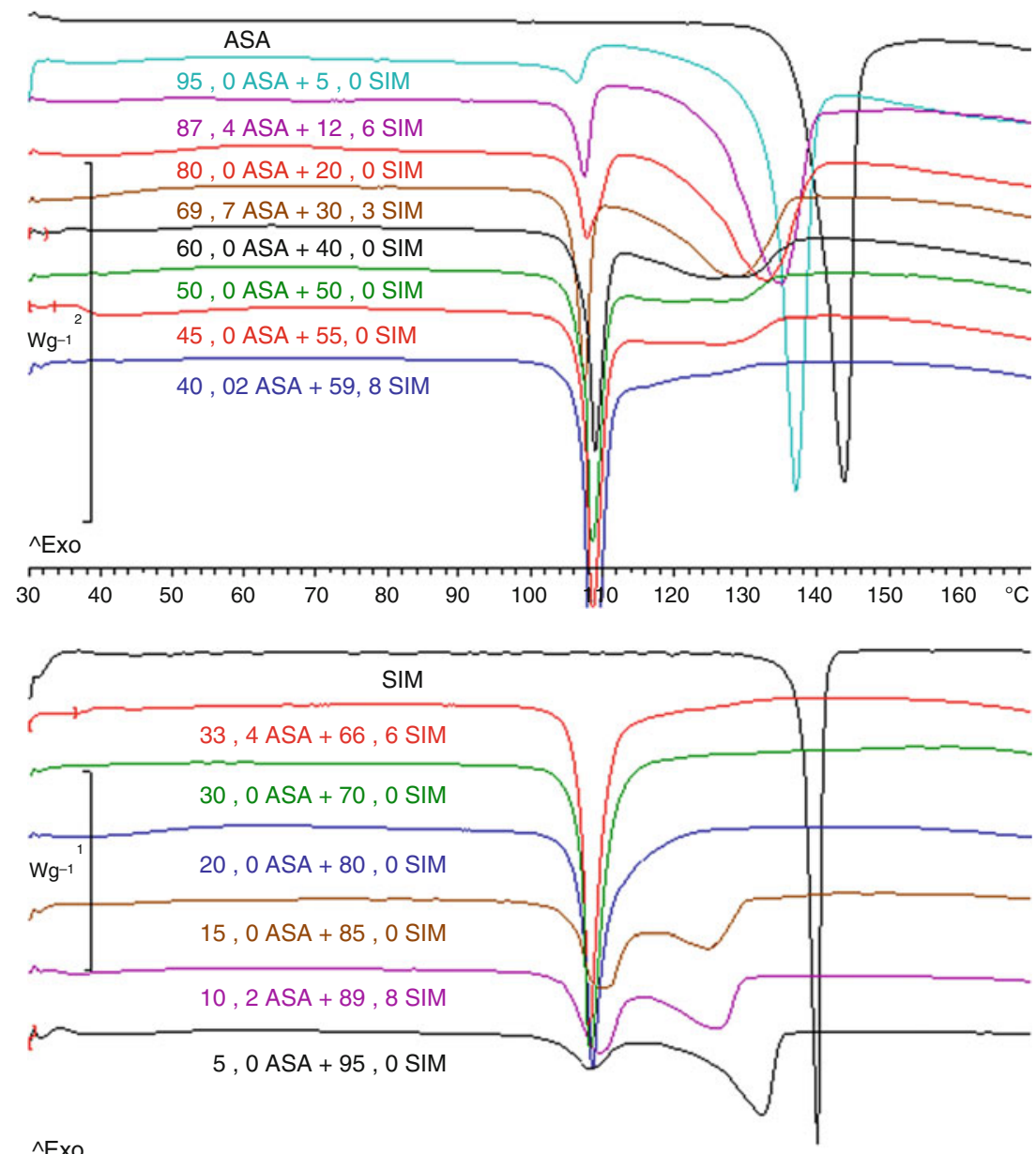

$\wedge$ Exo

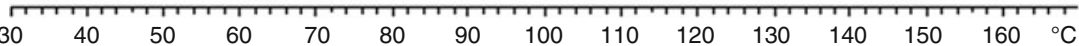

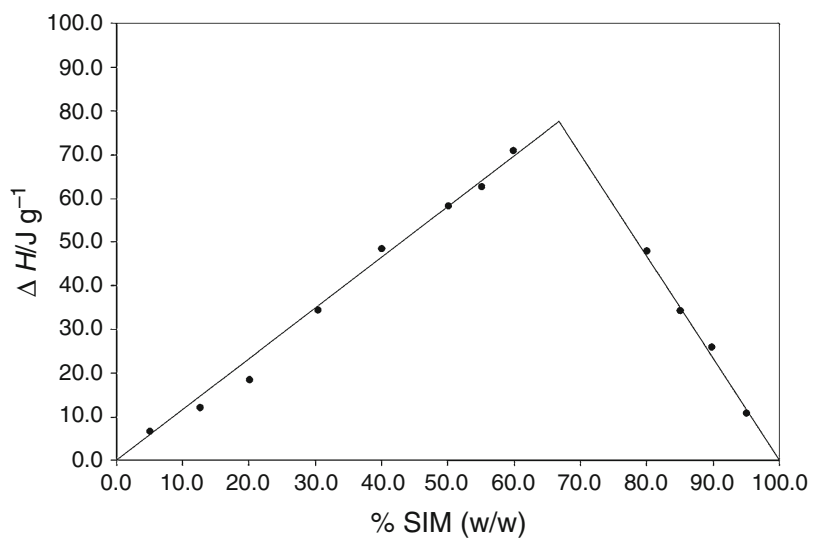

Fig. 4 Tamman's triangle construction for the eutectic melting enthalpy $\Delta H$ at $105.6^{\circ} \mathrm{C}$

\section{In vitro dissolution studies}

The dissolution profiles of the obtained mixtures of SIM with ASA in an aqueous solution containing $0.5 \%(\mathrm{w} / \mathrm{v})$ of SLS are shown in Figs. 7 and 8. The release rate profiles were plotted as a percentage of SIM or ASA dissolved

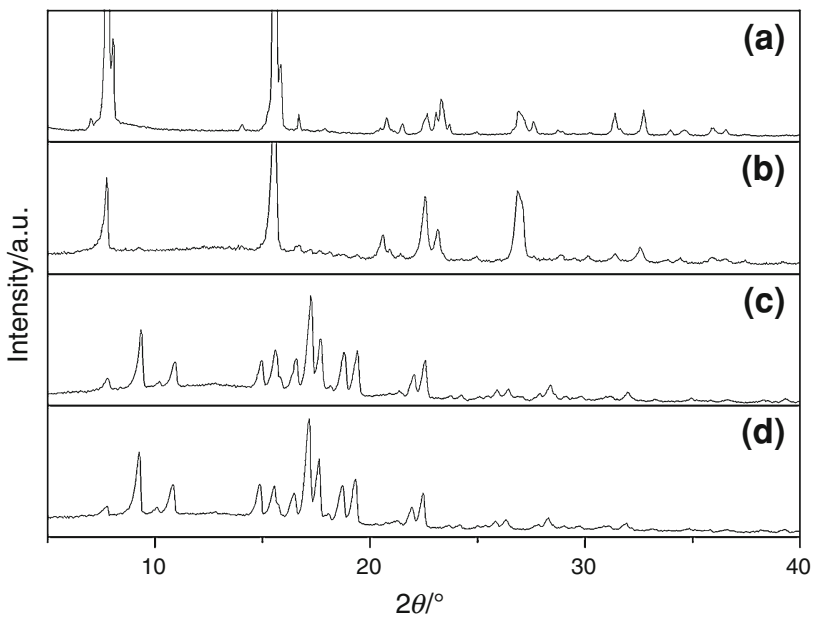

Fig. 5 XRPD patterns of ASA (a), 5.0/95.0 \% w/w SIM/ASA $(b)$, 95.0/5.0 \% w/w SIM/ASA $(c), \operatorname{SIM}(d)$

from the mixture and pure substance versus time. It was evident that the rate of dissolution of pure SIM was slow, less than $70 \%$ of the substance being dissolved within 60 min. With the two components combined, in the first 
Fig. 6 IR spectra of ASA $(a)$, 19.4/80.6\% w/w SIM/ASA (b), $89.8 / 10.2 \%$ w/w SIM/ASA (c), $\operatorname{SIM}(d)$
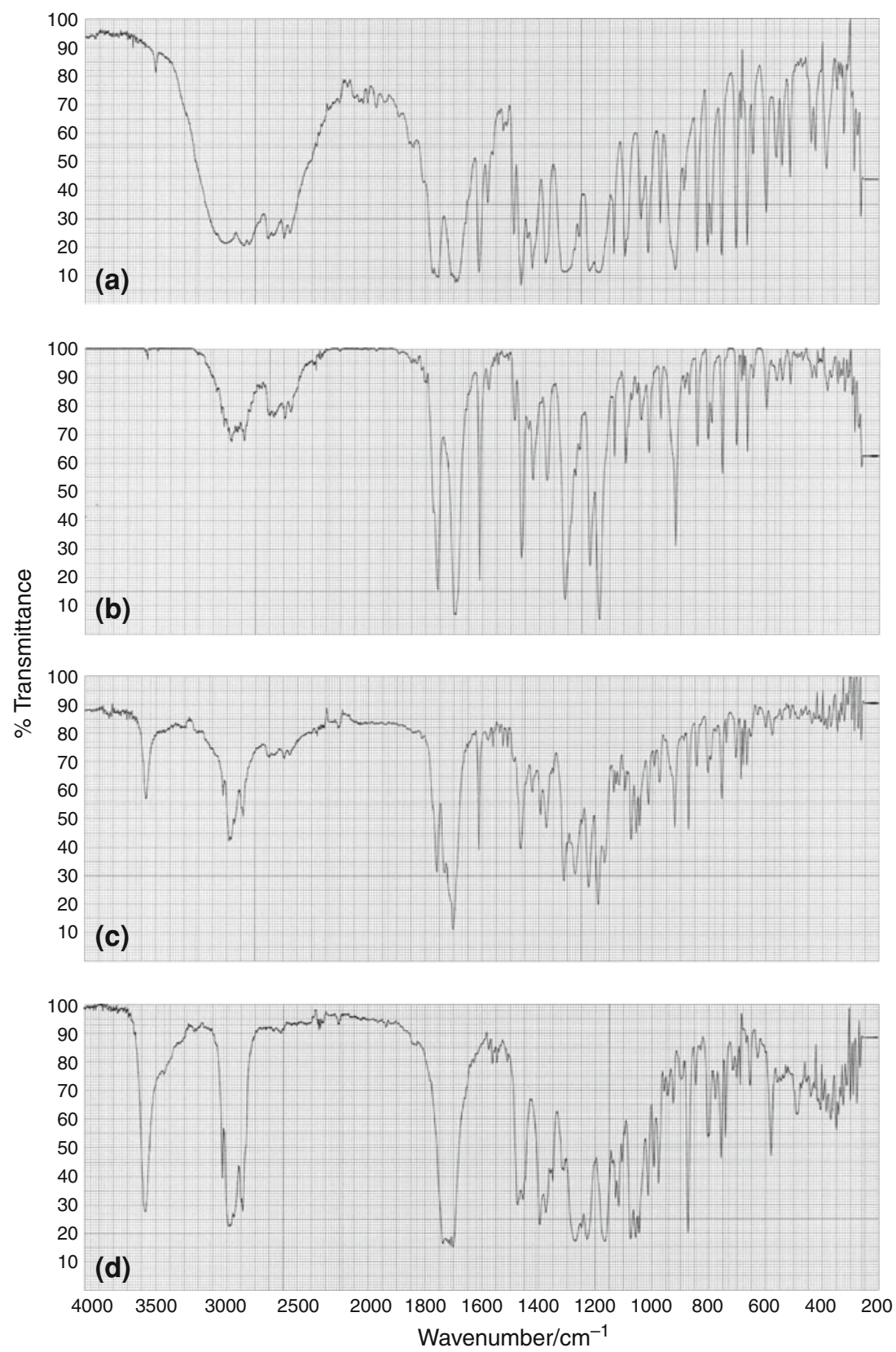

few minutes, SIM showed an increased rate of dissolution. Compared with the pure SIM, the dissolution rate of SIM from its mixtures appeared faster. It was $100 \%$ at the eutectic composition (66.6/33.4\% w/w SIM/ASA), which was 1.5 times versus SIM alone.

The reason for the improved dissolving capacity of eutectic mixtures of the components is the production of crystals of a suitable size, increasing their surface dissolution, inhibiting the formation of agglomerates of pure components, and increasing the wettability of the substance. For the dispersions containing $85.0 \%, 80.0 \%$,
$89.8 \% \mathrm{w} / \mathrm{w}$ SIM, the noticeable solubility is weaker. Probably, the progress of dissolution was determined by the low content of ASA, which improves the solubility of SIM and causes it to move more quickly to the solution.

Given the size of therapeutic doses of both substances, aspirin used in diseases of the cardiovascular system in the range of $75-100 \mathrm{mg}$ and simvastatin $10-80 \mathrm{mg}$, it can be concluded that the results obtained permit the development of dosage forms with modified physical properties, containing both components in the desired therapeutic concentration ranges. 

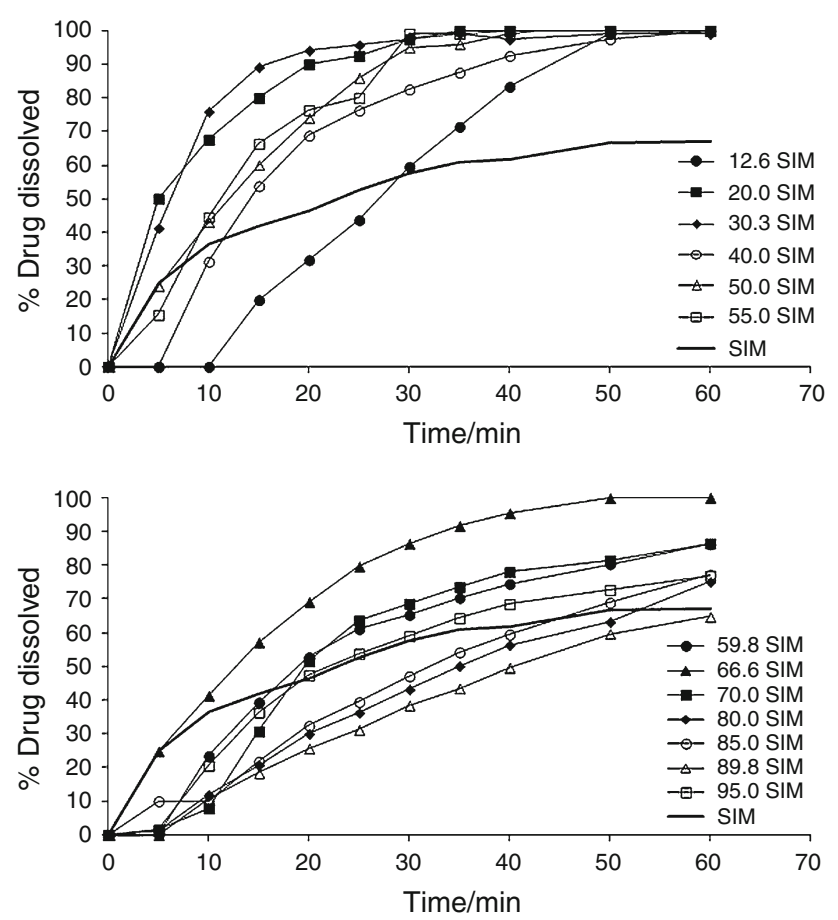

Fig. 7 Dissolution rate of SIM and its mixtures with ASA
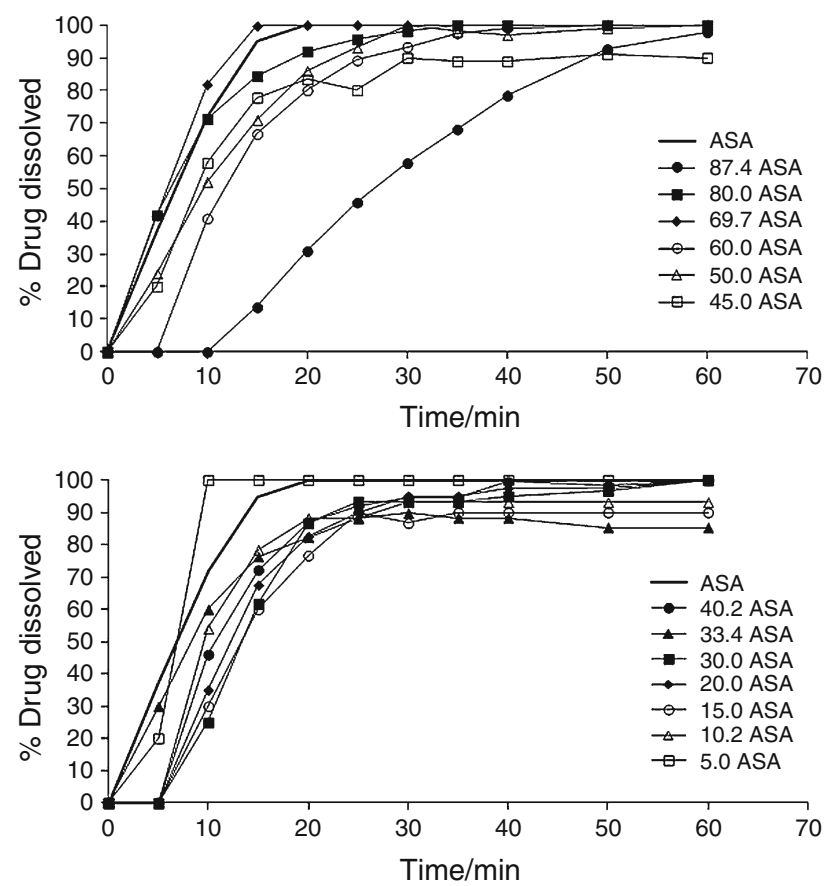

Fig. 8 Dissolution rate of ASA and its mixtures with SIM

\section{Conclusions}

Thermal properties of SIM-ASA physical mixtures were already investigated by Al-Suwayeh et al. [37], but the lowering of melting points of the components was not assigned to the eutectic formation. It is known that ASA forms eutectic mixtures with some crystalline compounds like acetaminophen and urea [38], propoxyphene $\mathrm{HCl}$ [39], phenobarbital [40], salicylic acid [41], and fenofibrate [42].

Our DSC studies clearly demonstrate the eutectic formation in SIM-ASA system, which significantly affects the dissolution of SIM in aqueous media. Dissolution enhancement of SIM was attributed to the effective wetting of the reduced drug particles released from eutectic composition. IR spectroscopy and XRPD studies of SIM-ASA mixtures showed no interaction between the drugs in a solid state. Our investigation indicates that the obtained SIM-ASA eutectic mixture can be applied for the preparation of a multi-component oral dosage form with improved dissolution of SIM and may have the potential to cause bioavailability improvements and consequently therapeutic efficacy.

Open Access This article is distributed under the terms of the Creative Commons Attribution License which permits any use, distribution, and reproduction in any medium, provided the original author(s) and the source are credited.

\section{References}

1. Seedher N, Sharma P. Solubility and stability enhancement of poorly-soluble drugs clarithromycin and prednisolone by combination with other drugs. Int J Biol Chem. 2007;1:229-36.

2. Mihos CG, Salas MJ, Santana O. The pleiotropic effects of the hydroxy-methyl-glutaryl-CoA reductase inhibitors in cardiovascular disease: a comprehensive review. Cardiol Rev. 2010;18: 298-304.

3. Tandon V, Bano G, Khajuria V, Parihar A, Gupta S. Pleiotropic effects of statins. Indian J Pharmacol. 2005;37:77-85.

4. Davignon J. Beneficial cardiovascular pleiotropic effects of statins. Circulation. 2004;109:III39-40.

5. Liao JK, Laufs U. Pleiotropic effects of statins. Annu Rev Pharmacol Toxicol. 2005;45:89-118.

6. Schneider MP, Bernhard M, Schmidt BM, John S, Schmieder RE. Effects of statin treatment on endothelial function, oxidative stress and inflammation in patients with arterial hypertension and normal cholesterol levels. J Hypertens. 2011;29:1757-64.

7. Awtry E, Loscazlo J. Aspirin. Circulation. 2000;101:1206-18.

8. Van Gijn J, Algra A. Aspirin and stroke prevention. Thromb Res. 2003;110:349-53.

9. Tirnaksiz E, Pamukcu B, Oflaz H, Nisanci Y. Effect of high dose statin therapy on platelet function; statins reduce aspirin resistant platelet aggregation in patients with coronary heart disease. J Thromb Thrombolysis. 2009;27:24-8.

10. Hennekens CH, Sacks FM, Tonkin A, Jukema JW, Byington RP, Pitt B, Berry DA, Berry SM, Ford NF, Walker AJ, Natarajan K, Sheng-Lin C, Fiedorek FT, Belder R. Additive benefits of pravastatin and aspirin to decrease risks of cardiovascular disease: randomized and observational comparisons of secondary prevention trials and their meta-analyses. Arch Intern Med. 2004;164:40-4.

11. Fisher M, Cushman M, Knappertz V, Howard G. An assessment of the joint associations of aspirin and statin use with $\mathrm{C}$-reactive protein concentration. Am Heart J. 2008;156:106-11.

12. Hassan HM, El-Gayar AM, Ibrahim TM, Al-Gayyar MMH. Antiinflammatory effect of simvastatin-aspirin combination. Int $\mathrm{J}$ Pharm Sci Drug Res. 2011;3:89-96. 
13. Amidon GL, Lennernäs H, Shah VP, Crison JR. A theoretical basis for a biopharmaceutic drug classification: the correlation of in vitro drug product dissolution and in vivo bioavailability. Pharm Res. 1995;12:413-20.

14. Joshi HN, Fakes MG, Serajuddin ATM. Differentiation of 3-hydroxy-3-methylglutaryl-coenzyme A reductase inhibitors by their relative lipophilicity. Pharm Pharmacol Commun. 1999;5: 269-71.

15. Ellison DK, Moore WD, Petts CR. Simvastatin. In: Brittain HG, editor. Analytical profiles of drug substances and excipients. San Diego: Academic Press Inc.; 1993. p. 359-88.

16. Schachter M. Chemical, pharmacokinetic and pharmacodynamic properties of statins: an update. Fundam Clin Pharm. 2004;19: $117-25$.

17. Varshosaz J, Tavakoli N, Salamat FA. Enhanced dissolution rate of simvastatin using spherical crystallization technique. Pharm Dev Technol. 2011;16:529-35.

18. Silva TD, Arantes VT, Resende JALC, Speziali NL, de Oliveira RB, Vianna-Soares CD. Preparation and characterization of solid dispersion of simvastatin. Drug Dev Ind Pharm. 2010;36: 1348-55.

19. Mandal D, Ojha PK, Nandy BC, Ghosh LK. Effect of carriers on solid dispersions of simvastatin (Sim): physico-chemical characterizations and dissolution studies. Der Pharm Lett. 2010;2: 47-56.

20. Patel R, Patel M. Preparation, characterization, and dissolution behavior of a solid dispersion of simvastatin with polyethylene glycol 4000 and polyvinylpyrrolidone K30. J Disper Sci Technol. 2008;2:193-204.

21. Rao M, Mandage Y, Khole I, Munjapara G. Characterization of solid dispersions of simvastatin with PVP K30 and poloxamer 188. Ind J Pharm Educ Res. 2011;45:145-52.

22. Süle A, Csempesz F. Complexation of statins with $\beta$-cyclodextrin in solutions of small molecular additives and macromolecular colloids. Progr Colloid Polym Sci. 2008;135:93-100.

23. Jun SW, Kim M-S, Kim J-S, Park HJ, Lee S, Woo J-S, Hwang S-J. Preparation and characterization of simvastatin/hydroxypropyl- $\beta$-cyclodextrin inclusion complex using supercritical antisolvent (SAS) process. Eur J Pharm Biopharm. 2007;66: 413-21.

24. Vasconcelos T, Sarmento B, Costa P. Solid dispersions as strategy to improve oral bioavailability of poor water soluble drugs. Drug Discov Today. 2007;12:1068-75.

25. Verma S, Rawat A, Kaul M, Saini S. Solid dispersion: a strategy for solubility enhancement. Int J Pharm Technol. 2011;3: 1062-99.

26. Yuan X, Capomacchia AC. The binary eutectic of NSAIDs and two-phase liquid system for enhanced membrane permeation. Pharm Dev Technol. 2005;1:1-10.
27. Yuan X, Capomacchia AC. Physicochemical studies of binary eutectic of ibuprofen and ketoprofen for enhanced transdermal drug delivery. Drug Dev Ind Pharm. 2010;36:1168-76.

28. Lin D, Huang Y. A thermal analysis method to predict the complete phase diagram of drug-polymer solid dispersions. Int $\mathrm{J}$ Pharm. 2010;399:109-15.

29. Szterner P, Legendre B, Sghaier M. Thermodynamic properties of polymorphic forms of theophylline, Part I: DSC, TG, X-ray study. J Therm Anal Calorim. 2010;99:325-35.

30. Gilchrist SE, Letchford K, Burt HM. The solid-state characterization of fusidic acid. Int J Pharm. 2012;422:245-53.

31. Aignera Z, Berkesib O, Farkasa G, Szabó-Révésza P. DSC, X-ray and FTIR studies of a gemfibrozil/dimethyl- $\beta$-cyclodextrin inclusion complex produced by co-grinding. J Pharm Biomed Anal. 2012;57:62-7.

32. Basu P, Alexander KS, Riga A. A statistical model for the optimization of DSC performance in the evaluation of drugs for preformulation studies. J Therm Anal Calorim. 2006;83:19-22.

33. Oliveira PR, Bernardi LS, Murakami FS, Mendes C, Silva MAS. Thermal characterization and compatibility studies of norfloxacin for development of extended release tablets. J Therm Anal Calorim. 2009;97:741-5.

34. Höhne GWH, Hemmninger W, Flammersheim H-J. Differential scanning calorimetry. Berlin: Springer; 1996.

35. Gabott P. Principles and applications of thermal analysis. Oxford: Blackwell Publishing; 2008.

36. Polish Pharmacopoeia VIII. Warsaw: Ministry of Health; 2008.

37. Al-Suwayeh SA, Ahmed MO, Mahrous GM, Taha EI. Preparation and in vitro evaluation of tablets containing triple drug combination used for prevention/treatment of cardiovascular diseases. Aust J Basic Appl Sci. 2009;3:3684-91.

38. El-Banna HM. Solid dispersion of pharmaceutical ternary systems I: phase diagram of aspirin-acetaminophen-urea system. J Pharm Sci. 1978;67:1109-11.

39. Law SL, Lo WY, Lin FM, Chiang CH. Compatibility study of propoxyphene $\mathrm{HCl}$ solid mixtures using differential scanning calorimetry. Drug Dev Ind Pharm. 1988;14:1465-70.

40. Sangster J. Phase diagrams and thermodynamic properties of binary systems of drugs. J Phys Chem Ref Data. 1999;28: 889-930.

41. Campanella L, Micieli V, Tomassetti M, Vecchio S. Solid-liquid phase diagrams of binary mixtures. Acetylsalicylic acid(1) + E(2) (E 5 salicylic acid, polyethylene glycol 4000, D-mannitol). J Therm Anal Calorim. 2010;99:887-92.

42. Górniak A, Wojakowska A, Karolewicz B, Pluta J. Phase diagram and dissolution studies of the fenofibrate-acetylsalicylic acid system. J Therm Anal Calorim. 2011;104:1195-200. 